\title{
QUADERNI Quaderni
}

Communication, technologies, pouvoir

\section{8 | Automne 2015}

\section{Les séries, politique fiction}

\section{House of Cards. Qu'est-ce qu'un coup politique fictionnel?}

House of Cards: How Far Fiction Can Depict Political Moves?

Emmanuel Taïeb

\section{OpenEdition}

1 Journals

\section{Édition électronique}

URL : https://journals.openedition.org/quaderni/923

DOI : 10.4000/quaderni.923

ISSN : 2105-2956

\section{Éditeur}

Les éditions de la Maison des sciences de l'Homme

\section{Édition imprimée}

Date de publication : 5 octobre 2015

Pagination : 67-81

\section{Référence électronique}

Emmanuel Taïeb, « House of Cards. Qu'est-ce qu'un coup politique fictionnel ? », Quaderni [En ligne], 88 । Automne 2015, mis en ligne le 05 octobre 2017, consulté le 28 juin 2022. URL : http:// journals.openedition.org/quaderni/923; DOI : https://doi.org/10.4000/quaderni.923 


\section{$D$ ossier}

\section{House of Cards Qu'est-ce qu'un coup politique fictionnel?}

\section{Emmanuel \\ Taïeb}

Institut Universitaire de France Sciences Po Lyon / Triangle Reason. Backed up by murder. " Kay Adams, Le Parrain III (1990)
« That's your big thing, isn't it, Michael?

Le « coup » est l'atome de l'activité politique, l'occupation principale des professionnels de la politique. C'est aussi la raison d'être et l'objet de la science politique, à partir du moment où elle a entrepris de saisir tout ce que le jeu politique devait à l'action même des titulaires de rôles, et non aux seules règles de droit. Le coup joué témoigne de la capacité des acteurs à saisir les normes comme à s'en affranchir, à s'appuyer sur leurs capitaux et ressources, pas seulement institutionnels, pour sortir vainqueurs de divers rapports de force ${ }^{1}$. La stratégie politique, nécessairement fluctuante et relationnelle, s'appuie sur des séries de coups joués simultanément et successivement par tous ceux qui agissent dans le champ politique. Du refus d'un président de la République de signer les projets d'ordonnances d'un gouvernement de cohabitation, aux innombrables coups joués quotidiennement (poser une question à la Chambre, procéder à une nomination, participer à une émission de télévision, mobiliser des soutiens, saisir une haute cour pouvant bloquer le processus législatif, etc.), la lutte politique est structurée par toutes les initiatives visant à s'assurer du maintien d'une position dominante ou gratifiante, à la reconquérir, et au passage, le plus souvent, à préserver l'ordre politique même qui conditionne l'existence de cette position.

L'univers politique incarne aussi, dans les sociétés démocratiques, le dépassement de la violence physique, et sa conversion par les hommes politiques en une violence symbolique. Laquelle 
implique d'autres armes. Celle de l'élection, qui confère le droit de s'exprimer au nom d'un plus grand nombre. Celle du poste, qui permet d'activer divers leviers décisifs. Ou celle de la rhétorique, où l'on « tue » autrement son adversaire. C'est là que se trouve la dimension « dramatique » du politique, qui la transforme en une évidente matière fictionnelle, dont la dernière incarnation se trouve dans plusieurs séries dont les personnages appartiennent au champ politique ou à ses «champs adventices ${ }^{2}$ » ( $A$ la Maison-Blanche, Borgen, Boss, Commander in Chief, The Wire, etc.). Si ces programmes ont rapidement attiré l'attention des chercheurs en sciences sociales, c'est précisément parce qu'ils semblaient donner à voir le « réel », le « terrain », accessible et mis en récit dans une forme idéale. Au risque cependant de basculer dans une « sofa sociology $y^{3}$ », dans l'illusion de croire que le réalisme se confondrait avec la réalité, et dans l'oubli que tout ce qui est montré renvoie à des intentions, des conditions de production, des scénarios, et demeure irréductiblement fictionnel. Ne voir que ce qui semble réaliste dans une série, reviendrait à transformer ce support en un simple enregistrement du réel, à écraser ce qui fait sa singularité narrative et sa volonté artistique de livrer autre chose que du réel brut. Cette volonté peut bien sûr s'appuyer sur un intérêt pour des thèmes sociologiques, mais sans se confondre avec une démarche scientifique. On sera au mieux dans une « social science fiction ${ }^{4}$ », une œuvre de fiction qui délivre une certaine forme de "vérité », et stimule l'imagination sociologique du spectateur, mais sans ambition de dire le vrai.

Paradoxalement, la familiarité de la fiction la fait tenir pour réelle, alors qu'à l'inverse le réel peut paraître étrange et appelle une explication. "C'est le monde fictionnel qui est attendu, cohérent, connaissable, partagé, et c'est la réalité qui reste lointaine, incertaine et problématique », écrit ainsi Sabine Chalvon-Demersay ${ }^{5}$. Car la fiction est une mimèsis construite par des procédés techniques et des effets, une ressemblance intentionnelle, le « faire-comme-si » dans les arts représentationnels, dont parle Jean-Marie Schaeffer $^{6}$. Une démarche heuristiquement féconde consiste alors à s'intéresser à la part fictionnelle de la fiction. Aborder une série, c'est travailler ce qui dans la fiction relève purement du fictionnel, puis du plausible, puis du réaliste, et travailler l'écart entre ces éléments, pour chacun les éclairer.

House of Cards est une série sans doute moins inventive que plusieurs de ses contemporaines ${ }^{7}$, mais, en dépit de son réalisme apparent, de son ambition de montrer les coulisses peu reluisantes du métier politique, elle entretient le même rapport au fictionnel que les séries de sa génération. C'est à cet égard que son analyse avec les outils des sciences humaines est intéressante, car elle permet de penser la pratique et la notion de coup politique à partir de leurs représentations fictionnelles, à la fois puissantes et exagérées, en tout cas révélatrices d'une certaine perception du monde politique contemporain ; quelque part entre Machiavel et Shakespeare, qui sont les noms qui reviennent souvent pour évoquer la série ${ }^{8}$. Or, c'est justement dans l'ambiguïté entre activité politique et activité criminelle, entre série politique et thriller, que House of Cards tire à sa limite la notion de coup politique, en transgressant la règle qui veut que le politique soit précisément la fin du meurtre. Il faudra ainsi analyser comment cette série décrit le champ 
politique central américain, celui de Washington, et la manière dont son personnage principal, Francis « Frank » Underwood, use de tout ce que sa fonction lui permet de faire, et au-delà surtout, pour poursuivre ses intérêts ; comment la panoplie des coups qu'il joue glisse du plausible au fictionnel ; et comment enfin le basculement définitif dans la fiction s'opère quand les coups joués ne sont plus politiques mais au contraire dépolitisés. Il ne s'agit pas visiblement pour les créateurs de la série de montrer le jeu politique dans sa plausibilité, comme avait pu le faire $\grave{A} l a$ Maison-Blanche, mais de l'utiliser comme lieu pour sonder les noirceurs de l'âme humaine.

\section{Joueurs et cartes politiques}

House of Cards met en scène une figure politique centrale dans le système de Westminster, et dans une moindre mesure dans le régime présidentiel américain ${ }^{9}$, le Whip ou Chief Whip, qui est chargé de s'assurer du bon vote des parlementaires, du bon suivi des consignes du parti, et qui peut distribuer blâmes et gratifications. Même si aux ÉtatsUnis le parti du président peut être majoritaire à la Chambre des représentants, le résultat d'un vote n'est jamais acquis, car les congressistes disposent d'une autonomie partisane relative. Le whip est donc au cœur des relations triangulaires entre la Maison-Blanche, le Congrès et le parti, pour assurer une unité d'action; fusionnant les fonctions de chef de la majorité parlementaire et de ministre chargé des Relations avec le parlement, dans le système français. Le terme de whip (littéralement « fouet») vient originellement de la vénerie, où il désigne le « piqueur », du nom du valet à cheval qui règle la course des chiens à la chasse à courre ${ }^{10}$.
Le personnage d'Underwood contrôle donc peu ou prou l'appareil partisan, tel qu'il existe à la Chambre, et incarne cette direction professionnelle du parti dont parle Roberto Michels ${ }^{11}$. C'est un personnage dont la position au contact des différentes institutions de pouvoir lui permet d'être un relai puissant des orientations politiques du parti ou de la présidence, y compris en forçant la main des députés récalcitrants. C'est donc une personnalité redoutée, et dès le premier épisode, il se présente comme celui qui prend en charge les choses désagréables mais nécessaires, et s'occupe du « sale boulot», de la cuisine politicienne habituellement cachée. Le choix de Kevin Spacey comme acteur est ici intéressant, car son visage avenant, mais un peu quelconque par rapport à des acteurs hollywoodiens plus photogéniques, lui a permis de jouer des personnages duplices cachant de noirs desseins sous une apparence neutre (Usual Suspects, Minuit dans le jardin du bien et du mal), dans la lignée desquels Underwood s'inscrit.

Si la série, comme sa principale devancière $A$ la Maison-Blanche, est une plongée dans les coulisses de la politique, sa focalisation sur un personnage central - là où la série d'Aaron Sorkin jouait du côté « choral»- permet d'explorer minutieusement le métier politique et la notion de « rôle » politique. Underwood est un homme politique professionnel, membre du Parti démocrate, et élu chevronné, ayant passé 22 ans à la Chambre. Il se décrit lui-même comme un vieux briscard qui connaît le milieu et ses ficelles. L'idée d'une ascension politique liée à une revanche sociale est, au passage, discrètement suggérée, lorsqu'on apprend que son père était producteur de pêches et sa mère femme de 
ménage. Mais si certains épisodes font des allusions au passé du personnage, il est essentiellement pris dans son activité du moment. Précisément, comme d'autres séries américaines contemporaines (Les Experts, Dr House, Mad Men, etc.), House of Cards montre le monde du travail et sa dimension sacerdotale. On filme l'exercice d'un métier ${ }^{12}$, avec ses pratiques, ses mœurs, son vocabulaire, et ses objets. Si rituellement nombre de fins d'épisodes se déroulent dans l'appartement cosy des Underwood, l'impossibilité d'une vie personnelle où le travail n'entrerait pas est très souvent soulignée; le métier politique ne faisant pas ici exception. Éprouver des sentiments personnels risque de nuire à la vocation, d'obscurcir le jugement et d'entraver l'efficacité des coups. « Le cour peut étrangler l'esprit quand tout le sang s'y accumule. », déclare ainsi Underwood à propos d'un lobbyiste amoureux de Jackie Sharpe, la nouvelle whip qui lui a succédé (2x11).

« Avoir la connaissance pratique de ce qu'il convient de faire quand on est appelé à s'insérer dans des interactions successives en y occupant la même position (...), et être éventuellement capable d'expliquer pourquoi on agit ainsi, c'est être en mesure d'entrer dans "son" rôle. ", écrivait Jacques Lagroye à propos du professionnel de la politique ${ }^{13}$. Cette réflexivité de l'acteur politique sur son activité est matérialisée dans House of Cards par des regards-caméra exclusifs du personnage principal, où il décrit son état intérieur, les enjeux de la situation, ce qu'il s'apprête à faire, ou annonce les réactions des autres protagonistes. Ces adresses frontales au spectateur fonctionnent à son intention comme un « watch and learn », transformant la série en une « démonstration » par un maître, en un déploiement du savoir-faire de l'homme politique devenu insider. Le personnage ne fait pas que prendre à témoin le spectateur, il se fait guide dans le champ politique, pour l'inviter à tirer « quelque chose » de cette plongée (sur le « pouvoir», sur la « nature humaine », sur ce que l'homme fait à l'homme, etc.).

Dans tous les épisodes, Underwood montre sa capacité à endosser son rôle, en s'adaptant aux différentes configurations et interlocuteurs rencontrés. Froid et sombre à Washington, il s'habille d'un costume clair dans sa circonscription, et joue sur la corde émotionnelle dans un discours devant ses administrés, qui lui permet de retourner une situation où il était mis en cause. Cette faculté d'adaptation nécessite un travail particulier, qui n'est pas occulté par la série. C'est ainsi qu'on voit Underwood s'entraîner chez lui pour un lancer de balle de base-ball inaugural, car il en avait raté un des années auparavant (2x6). Un autre épisode suggère qu'un même rôle peut être tenu de manière différenciée, en s'attachant à la figure de Jackie Sharpe qui se montre une redoutable whip, en s'affranchissant des méthodes d'Underwood $(2 \times 4)$. Les autres facettes du métier politique - maîtrise des règles, surveillance des adversaires et anticipation de leurs coups, capitaux et privilèges de fonction permettant d'accéder à de nombreuses informations $^{14}$, veille médiatique, compréhension immédiate des entorses signifiantes aux règles (comme la secrétaire générale de la Maison Blanche se déplaçant au Capitole pour le voir) - sont également montrées. Tout comme le don d'ubiquité d'un Underwood qui gère d'importantes négociations syndicales par téléphone, alors qu'il est dans sa circonscription. 
Dans un épisode singulier (1x8), où Underwood retourne dans son ancienne école militaire, il fait un discours improvisé, mal construit, mais sincère, devant les cadets, laissant penser que loin de Washington, dans un cadre extra-politique, il y a moins de contrôle et plus d'affects. À moins que ce ne soit comme toujours un « relâchement contrôlé du contrôle ${ }^{15}$ ». C'est ainsi que quelques changements discrets apparaissent quand Underwood devient vice-président. Il s'adresse plus directement au président, cherchant une connivence personnelle, se présentant en chemise devant lui, restant parfois assis quand le président parle. Le rappel des positions reste cependant permanent, et la mise en scène s'y attache. Au début de l'épisode 7 de la saison 2, il y a jeu sur le champ-contrechamp dans le Bureau ovale,

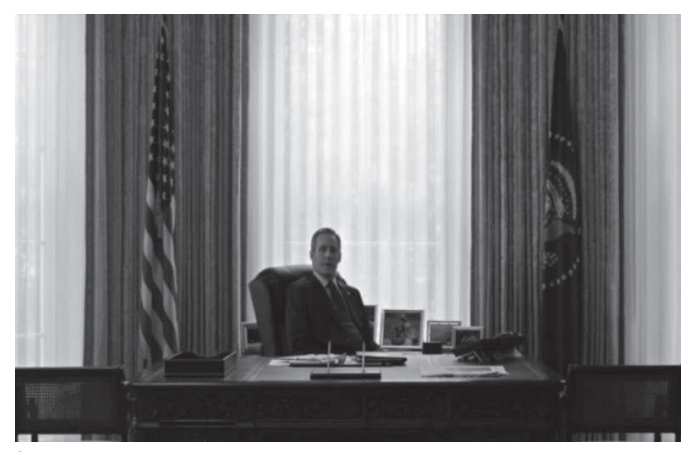

$2 \times 7$. Même s'il est du « bon » côté du bureau, le cadrage du président Walker le montre écrasé par la tâche.

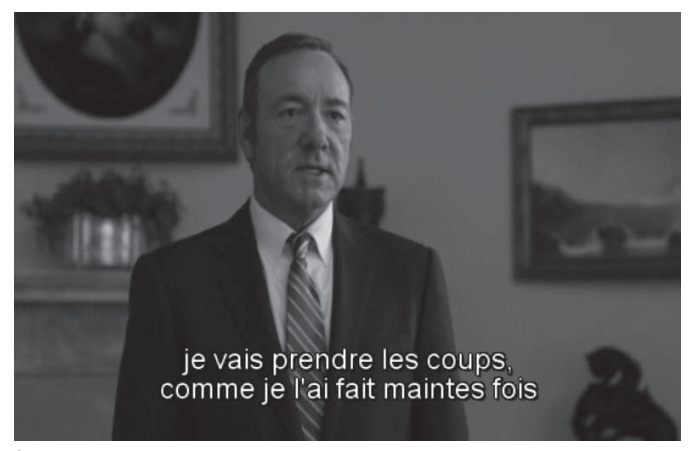

2x7. Underwood sermonné par le président Walker, du « mauvais » côté du bureau. comme si Underwood était du «mauvais côté » du bureau : c'est lui qui va prendre les coups assénés par le président. Il est cadré de près, mais sa rigidité montre sa résistance ; tandis que sur le contrechamp, on voit le président cadré en bas, comme écrasé par la tâche et les ors. À l'avantdernier épisode de la saison 2, Jackie Sharp est face à Underwood, assise dans un fauteuil à côté de son épouse, Claire, puis elle se lève, et Underwood se met à sa place; elle est face à eux, à la fois comme une enfant qu'on aiguillonne, mais aussi comme attendant l'annonce de la position intéressante qu'elle pourrait tenir dans le nouveau monde politique promis par Underwood s'il devenait président. Dans tous les cas, la série met en scène une activité politique faite de transactions et d'ajustements permanents des

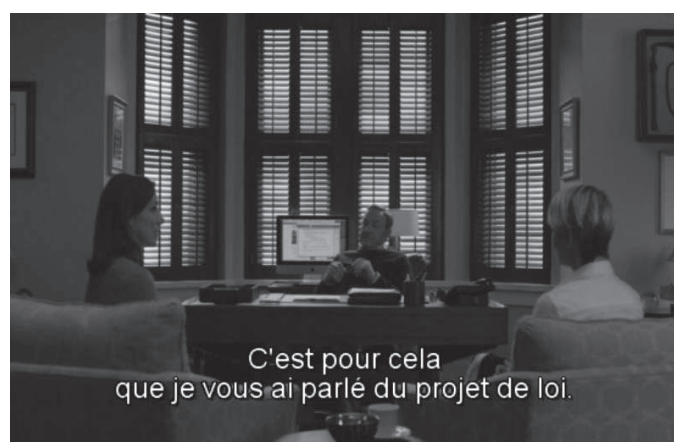

$2 \times 12$. Sharpe est mise dans la confidence de l'impeachment par les époux Underwood, présentés ici comme des mentors.

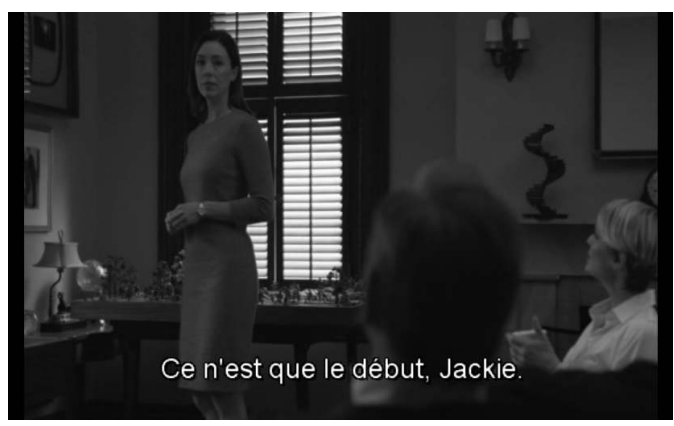

2x12. Une possible position dominante dans l'après-Walker. 
protagonistes, en fonction des prises de position des autres joueurs ${ }^{16}$, par la mobilisation d'un " "sens pratique" des prises de position possibles et impossibles, probables et improbables pour les différents occupants des différentes positions, [qui] permet de "choisir" les prises de position convenables », dans la lecture que fait Bourdieu du champ politique ${ }^{17}$.

\section{La distribution des cartes}

À côté de la capacité à « être du métier », l'autre grand enjeu politique sur lequel la série insiste est la distribution des postes, la lutte pour la « disposition des postes » qui, à côté de la lutte pour des « buts fondamentaux », rappelle Weber, est la fin de l'activité partisane ${ }^{18}$. Le poste garantit revenus et pouvoir à celui qui l'obtient, comme il garantit la loyauté de son titulaire à celui qui le donne. En permanence, House of Cards bruit des noms de candidats pressentis, de short lists, de changements et de promesses de postes. Underwood n'échappe pas à la règle, puisque l'argument même de la série est sa vengeance, lui à qui on avait promis le poste de secrétaire d'État, en récompense de son soutien au candidat élu président, le fade Garett Walker. Or, le fait qu'Underwood soit écarté au profit d'un autre le lance dans une mission inédite consistant dans un premier temps à se débarrasser de celui qui a obtenu ce poste, et dans un second temps à se débarrasser du président même qui n'a pas tenu parole. Underwood devient un homme de cour qui ne vit plus que pour sa revanche, à petits pas, tout en ne se départant pas de la posture de celui qui réagit bien à cette décision, et envoie des signaux d'humilité et de soutien à la décision du président. Ainsi, le whip dont la fonction était de faire jouer les autres, de les intéresser aux enjeux, va désormais jouer pour lui-même. C'est d'ailleurs ce qui lui permet d'avancer ses pions longtemps sans être démasqué, car l'option d'un jeu pour soi n'est pas immédiatement comprise par les partenaires-adversaires. Underwood n'agit plus qu'en fonction d'un agenda personnel caché, en décalage avec l'agenda parlementaire et présidentiel. De tels coups massifs, destinés à altérer en profondeur la configuration politique, ne peuvent se faire seulement selon les règles institutionnelles, car le pouvoir de décision du président rend cela impossible et ne peut être contesté directement (comme pouvait l'être le pouvoir du roi à la cour), donc il faut en passer par d'autres champs (dont le champ journalistique) et par des « coups tordus » et souterrains; à la fin du générique d'ailleurs, le drapeau américain renversé rappelle l'importance du dessous des cartes.

On mesure là toute la fidélité de la série au fonctionnement du champ politique (comme aux travaux de science politique qui l'ont pris pour objet). Mais l'apport propre de House of Cards est sa dimension fictionnelle, le trouble qu'elle instille entre activité politique et activité criminelle, la porosité qu'elle pose entre jeu politique pacifié et jeu politique violent. La toute première apparition du personnage le montre ainsi étranglant à mains nues, hors-cadre, un chien accidenté. Le ton est donné : cet homme est un tueur, littéralement, un chef mafieux. S'il peut tuer physiquement les animaux, il tuera physiquement, et pas seulement symboliquement, ses adversaires. Un peu plus tard, on verra Underwood jouer à un jeu vidéo sanglant. De manière plus appuyée, on apprend aussi qu'il mange de la viande au petit matin, et nombre de scènes le montrent 
attablé dans un restaurant spécialisé en grillades. Sa nature même est dépeinte comme celle d'un carnassier, qui n'hésitera pas à dévorer les faibles et les ennemis dans la jungle politique.

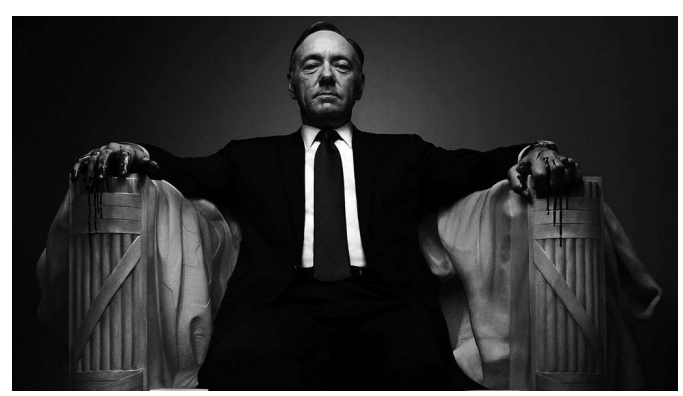

Affiche de promotion de la série, figurant Underwood, les mains ensanglantées, assis à la place de Lincoln au Lincoln Memorial à Washington.

Underwood est filmé comme un guerrier imparfaitement « curialisé $»^{19}$, qui refuse la pacification du jeu politique et l'abandon de la violence dans la compétition ${ }^{20}$. Certes, il est l'incarnation parfaite de l'homme de cour - à ceci près qu'en République le poste suprême n'est pas réservé au seul roi, et reste envisageable -, eliassien dans sa tactique, maîtrisant ses émotions, jouant d'alliances et de ruptures, manipulant, brillant par divers bons mots. Mais il sera également machiavélien dans sa stratégie, dans sa seule morale dans l'action $^{21}$ au nom des fins qu'il poursuit. Dans la lignée de Profit ${ }^{22}$, des Soprano, ou de Dexter, House of Cards met en scène un personnage malfaisant, antipathique ou arriviste, à propos duquel on vise moins l'identification mécanique du spectateur que l'exposition des « raisons » qui le font agir. Donc, non seulement la série s'inscrit dans cette tendance contemporaine à mettre en scène un « méchant ${ }^{23} »$, mais aussi à révéler son intériorité, pour explorer ici toute la part obscure du comportement politique.

Les séances de psychothérapie d'un Tony Soprano jouent ce rôle, tandis que dans House of Cards, ce sont les apartés face caméra d'Underwood : ce qu'il dit dans ces moments-là, et ce qu'on le voit entreprendre ensuite, participent du dévoilement de sa morale machiavélienne, et de la jubilation du spectateur à voir gagner celui qui est animé des plus mauvaises intentions. Cela sert également à déconstruire et expliciter le coup, en le resituant dans l'espace des possibles politiques (pour les spectateurs les moins familiers avec cet univers), et dans les modes de fonctionnement des autres acteurs ; montrant la nécessité de maîtriser leur psychologie, leurs trajectoires biographiques et leurs désirs, afin de les acculer politiquement ou de leur donner la gratification qu'ils attendent. Selon sa morale, Underwood considère que le jeu politique l'emporte sur le jeu institutionnel, que le principe démocratique, notamment l'élection, sont d'insupportables contraintes. Son accession à la vice-présidence est ainsi le seul moyen de potentiellement devenir président sans être préalablement élu. C'est aussi le sens de sa formule : « Democracy is so overrated. » $(2 \times 2)$.

\section{Bonnes et mauvaises mains}

Les épisodes de House of Cards sont en grande partie structurés par les coups politiques successifs que joue Underwood. Une typologie rapide permet de voir qu'il recourt à des coups de nature différente, la plupart prenant place dans le champ politique central. Par exemple, des coups défensifs : contrer les coups des adversaires, ou prononcer un discours de justification; et des 
coups proactifs : procéder à des inaugurations, faire des donations habilement ciblées, influencer et persuader par la parole, devenir informateur pour Zoe Barnes au Washington Herald afin de maîtriser une communication occulte, obtenir des votes favorables aux propositions de loi présentées (y compris, des votes républicains), promettre des postes, comme on l'a dit, être dans la démagogie (il apporte ainsi les reliefs d'un repas à des manifestants devant l'hôtel où des députés étaient reçus), imposer des éléments de langage pour influencer la lecture d'une situation (dans un épisode [1x6], Underwood lance l'expression « disorganised labour », qu'on va retrouver ensuite dans la bouche du speaker de la Chambre, dans celle d'un éditorialiste, etc.), acheter des loyautés (par exemple, celle de son garde du corps, renvoyé pour avoir tiré dans la rue, qu'Underwood réintègre à son service), jusqu'au coup le plus puissant : la menace d'une procédure d'impeachment contre le président, puis la nomination d'Underwood au poste de président. À côté, quelques autres coups prennent place dans le champ politique périphérique : éteindre un début d'incendie politique dans sa circonscription, $\mathrm{y}$ « tenir $»$ un candidat, recharger là sa légitimité politique.

Mais pour saisir la part fictionnelle et scénaristique de certains coups, il faut s'intéresser davantage à leur échelle qu'à leur nature ou leur centralité. Ainsi, à l'échelle macroscopique, Underwood joue une série de coups étalés - qui correspondent scénaristiquement à des arcs ; et un grand coup unique, qui est la conquête de la présidence - qui correspond scénaristiquement à ce qu'on appelle une «mythologie ${ }^{24} »$. C'est-à-dire un arc narratif qui se confond avec l'ensemble même des saisons, et en constitue le récit souterrain mais central. À cet égard, House of Cards obéit à l'écriture traditionnelle des séries télé, alternant stand alone episodes et épisodes où, à côté de l'intrigue principale, l'intrigue secondaire nourrit un arc ou une mythologie qui surdétermine toute la narration. Le « coup » est d'abord d'écriture avant d'être politique. Le constat est donc que plus l'intrigue explore la mythologie, plus les coups joués paraissent fictionnels. Dans l'écriture même du récit, plus le coup est important, moins il est plausible. La fiction est ainsi réactivée chaque fois qu'il faut donner une plus grande force de frappe au personnage principal. C'est ainsi que la manière dont, épisode après épisode, le piège autour du président se referme, semble cousu de fil blanc, avec un Walker qui s'enferre dans une thérapie secrète, prend des médicaments, nomme un procureur spécial, et apparaît absolument privé de soutiens ou de capacité à jouer ses propres coups. Fiction que cette tentative d'impeachment pour des faits peu sérieux, qui engloutit le président sans échappatoire possible.

C'est qu'Underwood fonde ses coups tantôt sur la règle de droit, et son bon respect (forcer les sénateurs républicains à siéger lors d'une discussion parlementaire importante), tantôt au contraire sur la subversion du droit par le politique, en s'affranchissant autant que possible des fonctionnements institutionnels : « Les règles ne font pas partie des choses que je tiens en haute estime. », dit-il (1x13). La série force donc le trait sur la « politique officieuse », celle qui se fait à hauteur d'homme, dans des relations interpersonnelles et clientélistes, ou dans des entrelacs d'intérêts varié $^{25}$. Elle met en scène la dimension purement 
politicienne de l'activité institutionnelle et du jeu des acteurs, leur capacité à s'écarter des règles, et le triomphe d'intérêts propres sur tout autre contingence ou ambition. Elle présente aussi certains coups comme des tentatives, plus ou moins maîtrisées, de peser sur la situation. Mais ni le contrôle du coup, ni celui de ses effets ne sont absolus, et les acteurs ne maîtrisent que partiellement leur propre action et celles des autres joueurs ${ }^{26}$. Underwood accepte ainsi de se mettre régulièrement en danger. Comme lorsqu'il s'exprime devant une assemblée hostile dans sa circonscription, ou quand il demande au père d'une jeune fille morte s'il veut qu'il démissionne (faisant peser sur lui un non-choix). À d'autres moments, il est même montré en grande difficulté, comme lorsqu'il perd pied dans un duel télévisé qu'il avait mal préparé, et que des images ridicules se répandent sur internet (1x6).

L'action l'emporte également sur les idées ou sur l'idéologie. " "En avant!" C'est notre cri de guerre. Je laisse l'idéologie aux généraux du dimanche. Ça ne me sert à rien. "), déclare Underwood (1x1). Ce qui compte est donc le seul jeu politique en vase clos, sans attention à ses fins, ou à ses programmes. À côté de la morale dans l'action, la série promeut donc une morale de l'action, au sens où l'action politique est première. Gagne politiquement celui qui agit sans arrêt, qui ne dort jamais, et double ainsi son temps de travail. L'idée que l'activité politique est une activité à temps plein est suggérée dès le générique, où la journée à Washington s'écoule en accéléré, y compris la nuit. À l'activité diurne d'Underwood s'ajoute ainsi une activité nocturne où, dans l'espace domestique, il met en perspective avec sa femme les événements écoulés. Ce qui est une autre manière de faire participer le spectateur à à la réflexivité sur le rôle politique. L'intimité du couple est habitée par le politique, en vertu du pacte implicite qui semble exister entre eux (Claire parle de « partnership » pour désigner leur relation), pour qu'Underwood puisse assouvir ses ambitions politiques, et sans que des écarts, adultérins par exemple, aient une quelconque incidence. La froideur du couple Underwood, son absence de repos (jogging au petit matin) les apparentent à des vampires, donc à des personnages éminemment fictionnels. En écho au régime carnivore d'Underwood, la série suggère que les deux individualités entrent en politique pour satisfaire leur amour du sang, et leur désir de mettre à mort les autres. Une séquence montre ainsi les époux attirés comme des requins par la blessure de Meechum, leur garde du corps. Claire fait renvoyer dix-huit personnes, et renvoie elle-même son adjointe, sans aucun état d'âme et sans marquer d'émotions. Ce sont des gens inhumains, condescendants à l'égard des plus faibles : ainsi du mépris de Claire pour une vieille et lente caissière, ou de celui d'Underwood pour un homme fou qui protestait devant le Congrès, auquel il dit que personne ne l'entend ni se soucie de lui.

L'argument de House of Cards est d'injecter des monstres dans le monde politique, ou de suggérer qu'il les produit, pour voir comment ils y évoluent. Le deuxième épisode flirte d'ailleurs avec le fantastique, via l'apparition d'une étrange vieille femme, qui sermonne Claire qui était allée courir dans un cimetière $(1 \times 2)$. À part quelques atmosphères nocturnes ou brumeuses, la présence du fantastique sera ensuite moins explicite, mais cela laisse penser qu'une forte inflexion 
scénaristique entendait dépeindre le monde politique comme un monde étrangement peuplé, dont le fonctionnement relève d'une « culture ésotérique $^{27}$ ». À l'image de sa perception par les profanes, que la série prend pour point de départ, sur le mode du dévoilement d'une activité perçue comme incompréhensible ou secrète, loin de sa nécessaire publicitée ${ }^{28}$. Mais à l'image aussi de sa perception par certains journalistes, qui font, postés derrière les grilles de la cage, la chronique du combat des « grands fauves ${ }^{29} »$. Un monde matérialiste, où seuls comptent les intérêts individuels. «I pray to myself for myself. », dit Underwood dans une église (1x13), avant, dans un autre épisode $(3 \times 4)$, de cracher sur une statue de Jésus. Cette monstruosité du couple Underwood signale qu'on est moins dans le réalisme politique que dans une fiction tentée par la dépolitisation.

\section{Joueurs et cartes dépolitisés}

La part fictionnelle de House of Cards se mesure au choix scénaristique de confondre politique et meurtre, et paradoxalement de dépolitiser ce qui devrait être des coups politiques. Cette présence de la fiction est marquée très vite, dès le générique, dont la musicalité pourrait être celle d'un thriller. La série penche à la fois du côté du genre policier et du film d'espionnage. Genre policier dans le choix des atmosphères : rendez-vous nocturnes et louches sous des ponts, dans des clubs ou des casinos ; mais sans qu'Underwood incarne jamais la figure du policier résolvant des énigmes, qui caractérise le genre $^{30}$. Film d'espionnage, parce que nombre de coups « politiques » sont en fait des barbouzeries. On est dans le « thriller politique », genre méconnu en France ${ }^{31}$. Pour les showrunners, les moyens du jeu politique incluent « naturellement » des actions viles, répréhensibles ou violentes. C'est ainsi que, dès le premier épisode, on filme des coups souterrains peu reluisants, plus ou moins crédibles, comme la fouille de poubelles par le directeur de cabinet d'Underwood, réduit ici au rôle de grognard. Missionné, le député Russo va récupérer des infos auprès d'une source en lui apportant une bouteille d'alcool et de la cocaïne. De même, on achète le silence de la prostituée qui était avec Russo le soir où il a été arrêté. La manipulation est permanente, y compris sexuellement. "Qu'a-t-elle à nous offrir ? », demande ainsi Claire à son mari quand elle apprend qu'il a couché avec la journaliste Zoe Barnes (1x5). Tout est chantage et pression. Ainsi d'une séquence un peu surréaliste où Underwood convoque deux députés hésitants de sa majorité, et les menace en sortant deux figurines qu'il fait tomber sur son bureau (1x9). Si de telles techniques existaient, les majorités parlementaires seraient facilement disciplinées...

Il y a du fictionnel aussi dans le fait qu'Underwood semble le seul politique roué (à l'exception de quelques rares interlocuteurs, notamment les lobbyistes), et le seul à avoir un répertoire de coups élargi, dans un monde où personne ne le voit venir, et où les autres ne semblent pas « jouer » mais juste subir le système. Est fictionnel l'emportement et les menaces de Russo contre Underwood quand son projet de loi ne passe pas, alors qu'il est évident qu'il subira des représailles qu'il n'est pas de taille à assumer. Le même Russo tombe bien naïvement dans le piège grossier que lui tendent Underwood et son adjoint Stamper : une vamp inconnue qui fait boire Russo et lui saute littéralement dessus, tandis qu'il lui 
cède sans aucune résistance, alors même qu'il pointait aux Alcooliques anonymes et avait axé toute sa campagne pour le gouvernorat sur sa renaissance après ses excès. On pourrait toujours plaider qu'Underwood tire son seul plaisir du jeu de massacre (instituer, destituer, manipuler) alors que les autres essaient de faire de la politique, il n'en reste pas moins que les scénarios lui donnent la part belle face à des adversaires plus faibles et manipulables via quelques leviers (fierté, amour-propre, faiblesses), y compris le président. Des adversaires surtout qui ne semblent jamais voir que les coups joués bénéficient toujours à Underwood. Certains acteurs font même des coups étranges. Est-il ainsi totalement crédible qu'un vice-président fasse campagne pour redevenir gouverneur de Pennsylvanie ? Lequel indique d'ailleurs qu'il ne démissionnera que s'il est élu gouverneur, sinon il restera vice-président ; mais quelle légitimité aurait un vice-président, pouvant potentiellement devenir président, s'il n'est même pas élu dans son État d'origine?

Pour le dire autrement, House of Cards est une série politique qui ne fait que dépolitiser les coups joués par les protagonistes. Ainsi de manière peu plausible, Underwood et Stamper se branchent sur la fréquence de la police, sans qu'on sache très bien ce qu'ils espèrent dans un premier temps. Une fusillade attire leur attention. Plus tard, on comprendra, quand Underwood passera chez Zoé Barnes pour lui dicter un tweet indiquant qu'un enfant de 8 ans a été tué alors qu'il aurait dû être à l'école, que c'est une manière de responsabiliser des enseignants grévistes. Par la suite, Underwood fait venir Spinella, solide leader des enseignants grévistes, dans une salle de réunion, le pousse à bout en l'insultant et en lui disant qu'il a mis en scène lui-même un lancer de briques contre son propre appartement, pour faire croire que c'était un acte commis par un gréviste en colère. Poussé à bout, Spinella le frappe violemment au visage. Underwood déclare alors qu'il ne portera pas plainte contre lui si la grève s'arrête immédiatement. On voit avec ces deux exemples combien certains coups gagnants joués par Underwood procèdent d'une dépolitisation et d'un déplacement au niveau personnel, sans que les rôles des acteurs entrent en ligne de compte. Comme si l'épuisement du répertoire de coups légitimes impliquait mécaniquement le recours à des coups illégitimes et extra-politiques, seuls capables d'avoir des effets marqués.

Le basculement définitif dans le fictionnel intervient quand Underwood tue lui-même Russo, qui, au passage, était mort politiquement et ne pouvait plus lui nuire. Puis quand, dans une séquence assez grotesque, un Underwood portant chapeau, manteau et lunettes pour ne pas être reconnu, précipite Zoe Barnes sous un métro. Il en va de même pour Stamper, dans tout le dernier épisode de la saison 3. En faisant d'un homme politique un meurtrier réel, et pas seulement un meurtrier symbolique, la série assume le mélange des genres, mais se renie elle-même comme série politique, sans avoir les moyens d'être une véritable série policière, ni d'espionnage. La saison 2 joue d'ailleurs moins de l'hybridité, et essaie de faire oublier ces meurtres originels, pour se concentrer uniquement sur d'habiles manœuvres purement politiques d'Underwood ; faisant alors ressembler la série davantage à $A$ la MaisonBlanche. Sauf que dans cette dernière série, les coups joués ne sont pas exorbitants du champ, mais tirent leur efficacité de leur originalité 
(quelque chose de jamais tenté), de leur astuce (faire dialoguer des acteurs issus d'un pays qui est en transition démocratique pour justement leur apprendre, à leur insu, les vertus du dialogue démocratique), ou de leur symbolique (le président Bartlet quittant la Chambre parce que le speaker le fait anormalement attendre ; il sort ainsi vainqueur du bras de fer, faisant passer l'attitude du speaker pour une marque d'irrespect du statut présidentiel). Là où $A$ la Maison-Blanche se voulait plausible, House of Cards ne saurait relever d'une pure analyse mimétique, tant le réel politique y apparaît en pointillés. Comme le conclut Michael Dobbs, pour évoquer son livre dont est tirée la série : "C'est une fiction, pas un documentaire. (...) En vérité, ce n'est pas une histoire de politique, c'est une histoire de condition humaine. ${ }^{32}$ »

Les créateurs de House of Cards n'ont pas eu confiance en la capacité du seul jeu politique à provoquer des situations dramatiques, et ont tenu à tirer la série vers d'autres genres et vers des formes plus classiques d'intrigues mêlant pouvoir et violence. Par là, ils contribuent à brouiller l'image du monde politique, en l'assimilant le plus souvent à un univers brutal, peuplé de gens sans émotion ni valeurs, où la vocation, l'intérêt général, les idées, les enjeux, les clivages, disparaissent derrière la seule volonté d'écraser les autres protagonistes. Si la peinture est documentée, le fictionnel demeure toujours à l'œuvre, aussi bien dans la théâtralité des postures et des dialogues, dans la lumière, que dans la capacité hors du commun du personnage principal à ne jamais perdre et à nuire. À ce titre, il n'est pas un homme politique mais un super-héros, une super figure du mal, entrée en politique.

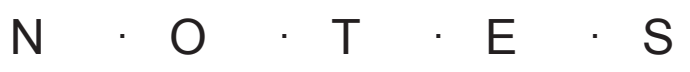

1. Bernard Lacroix, Jacques Lagroye (dir.), Le président de la République. Usages et genèses d'une institution, Paris, Presses de Sciences Po, 1992.

2. Michel Offerlé, Sociologie de la vie politique française, Paris, La Découverte, 2004, p. 42.

3. Fabien Truong, «"I've been schooled, dog. For real”. Apprendre et enseigner avec The Wire », in MarieHélène Bacqué, Amélie Flamand, Anne-Marie PaquetDeyris, Julien Talpin (dir.), The Wire. L'Amérique sur écoute, Paris, La Découverte, 2014, p. 212.

4. Ruth Penfold-Mounce, David Beer, Roger Burrows, "The Wire as Social Science-fiction? », Sociology, 45, 2011, pp. 152-167. http://soc.sagepub.com/ content/45/1/152

5. Sabine Chalvon-Demersay, « La part vivante des héros de séries », in Pascale Haag et Cyril Lemieux (dir.), Faire des sciences sociales. Critiquer, Paris, éd. de l'Ehess, 2012, p. 36.

6. Jean-Marie Schaeffer, Pourquoi la fiction?, Paris, Seuil, 1999, pp. 11 \& 13.

7. House of Cards est tiré d'un roman éponyme de Michael Dobbs, ancien conseiller de Margaret Thatcher, publié en 1989, et suivi de deux autres dans le même univers (To Play the King, The Final Cut). Avant la série américaine, on trouve une première adaptation britannique de la BBC, sous la forme d'une mini-série de quatre épisodes, inédite en France (1990). L'ouvrage est ensuite adapté aux États-Unis par Netflix en 2013, sous la direction du réalisateur David Fincher et du scénariste Beau Willimon. Nombre d'épisodes sont mis en scène par des réalisateurs chevronnés, ayant travaillé dans le cinéma (à commencer par David Fincher, ou James Foley et Joel Schumacher), réalisant souvent des films sombres, ce qui permet d'expliquer la tonalité générale de la série. À ce jour, on compte 
trois saisons et 39 « chapitres ». Pour mieux resituer les épisodes, nous avons opté pour des références indiquant la saison et l'épisode.

8. « 9 Things 'House Of Cards' Took From Shakespeare ", The Huffington Post, 21/02/2014. http://www.huffingtonpost.com/2014/02/21/house-ofcards-shakespeare-_n_4823200.html

9. Hanspeter Kriesi, Les démocraties occidentales. Une approche comparée, Paris, Economica, 1994 (chapitre 6).

10. Jean Gicquel et Pierre Avril, Lexique de droit constitutionnel, Paris, PUF, coll. " Que sais-je », $4^{e}$ éd. 2013, entrée «Whip »; Dictionnaire Littré en ligne : http://www.littre.org/definition/piqueur

11. Roberto Michels, Les partis politiques : essai sur les tendances oligarchiques des démocraties, Flammarion, 1971 [1914].

12. Charles Bosvieux-Onyekwelu, « L'Exercice de l'Etat : voyage dans le "back-office" de la politique », Quaderni, 86, Hiver 2014-2015, pp. 55-66.

13. Jacques Lagroye, «Être du métier », Politix, vol. 7, 28, $4^{\text {e }}$ trimestre 1994, p. 6.

14. Pierre Bourdieu, « La représentation politique. Éléments pour une théorie du champ politique », ARSS, 36-37, 1981, p. 19. Pour une synthèse des privilèges liés à la fonction de dirigeant, cf. Philippe

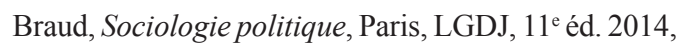
pp. 452-454.

15. Cas Wouters, Informalization. Manners \& Emotions since 1890, London, Sage Publications, 2007.

16. Murray Edelman, Pièces et règles du jeu politique, Paris, Seuil, 1991 [1988], p. 22.

17. Pierre Bourdieu, «La représentation politique », art. cité, p. 6.

18. Max Weber, Le savant et le politique, Paris, La découverte, 2003, p. 135.

19. Norbert Elias, La société de cour, Paris, Flammarion, 1995 [1969].
20. Daniel Gaxie, La démocratie représentative, Paris, Monchrestien, 2éd. 1996, pp. 12-13.

21. Quentin Skinner, Machiavel, Paris, Seuil, 1989 [1981].

22. Philippe Ortoli, «Profit : être au-delà », Cycnos, «Le méchant à l'écran : les paradoxes de l'indispensable figure du mal », vol. 29, 2, 2013.

23. François Jost, Les nouveaux méchants. Quand les séries américaines font bouger les lignes du Bien et du Mal, Paris, Bayard, 2015.

24. Typiquement, une série comme $X$-Files s'est appuyée sur ce procédé, illustrant, même dans des épisodes en apparence autonomes, sa mythologie principale du secret gouvernemental autour de la présence sur Terre d'extraterrestres. Sur cette forme d'écriture et ses effets, cf. Ursula Ganz-Blaettler, "Récits cumulatifs et arcs narratifs », in Sarah Sepulchre (dir.), Décoder les séries télévisées, Bruxelles, De Boeck, 2011. Sur le lexique propre aux séries, cf. « Le vocabulaire des séries TV : lexique » (29/09/2014) : http://www.cineseries-mag. $\mathrm{fr} /$ vocabulaire-series-tv-lexique/

25. Michel Offerlé, Sociologie de la vie politique française, op. cit., pp. 69-72.

26. Jacques Lagroye, «La légitimation», in Madeleine Grawitz, Jean Leca (dir.), Traité de science politique, volume 1, Paris, PUF, 1985, p. 463.

27. Pierre Bourdieu, «La représentation politique », art. cité, p. 9.

28. Habermas rappelait que l'activité de la cour royale était secrète, et que la publicité démocratique s'est précisément opposée à cette pratique. Cf. Jürgen Habermas, L'espace public. Archéologie de la publicité comme dimension constitutive de la société bourgeoise, Paris, Payot, 1997 [1962]. Sur les « profanes », Céline Braconnier, Jean-Yves Dormagen, La démocratie de l'abstention. Aux origines de la démobilisation en milieu populaire, Gallimard, coll. « Folio Actuel», 
2007. Enfin, pour une lecture ésotérique de Twin Peaks, cf. Pacôme Thiellement, La main gauche de David Lynch. Twin Peaks et la fin de la télévision, Paris, PUF, 2010.

29. On songera par exemple aux titres de certains documentaires politiques de Patrick Rotman : Chirac, le jeune loup (2006) ; Chirac, le vieux lion (2006) ; Les Fauves (2011).

30. Luc Boltanski, « Une étude en noir », Tracés, 20, 2011, pp. 49-73.

31. C'est d'ailleurs l'expression utilisée par l'éditeur français du roman, qu'il publie dans une collection intitulée « Thriller ». Michael Dobbs, House of Cards, Paris, Milady, 2014.

32. Pierre Boisson, « Obama et Clinton sont fans d'House of Cards », entretien avec Michael Dobbs, Society, 2, mars-avril 2015, p. 16.

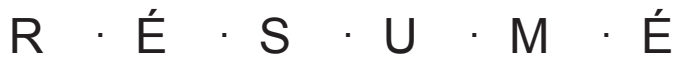

House of Cards (version américaine) joue explicitement de l'hybridité entre « thriller » et «politique». Cela permet de travailler la façon dont cette série décrit ou invente des « coups politiques », qui glissent du plausible au fictionnel, du genre politique au genre policier. L'article met en évidence un mouvement de basculement dans la fiction qui s'opère quand les coups joués ne sont plus politiques mais au contraire dépolitisés. Ce mouvement repose avant tout sur l'écriture propre de la série : plus l'intrigue explore l'arc narratif le plus large (l'ambition majeure du principal protagoniste, Frank Underwood, pour chaque saison), plus les coups sont fictionnels. Donc scénaristiquement, plus le coup est important pour la narration et moins il est plausible. La fiction est ainsi réactivée chaque fois qu'il faut donner une plus grande force de frappe au personnage principal, mais au détriment du réalisme politique.

\section{Abstract}

House of Cards: How Far Fiction Can Depict Political Moves?

House of cards (US version) explicitly explores the ambiguity between « thriller» and « politics ». This TV series offer a good starting point to study how fiction depicts various « political moves », going from plausible to fictional, from political genre to crime fiction. This paper highlights how the storyline dives into fiction, when the moves are no longer political and become depoliticized. This movement lies essentially in the very writing of the show: the more the plot explores the main narrative arc (at each season, the greatest ambition of the main protagonist, Frank Underwood), the more the actions become unrealistic. In other words, the script gains in narration what it 
loses in plausibility. That way though, the storyline is reactivated every time the main character needs to be placed in an empowering position. However, this is done at the expense of the political realism. 
